\title{
KINEMATICS OF FAINT STARS, AND THE REDUCTION FROM RELATIVE TO ABSOLUTE PROPER MOTION
}

\author{
C. A. Murray and S. V. M. Clube \\ Royal Greenwich Observatory
}

With the imminent completion of AGK3 in the northern hemisphere, and the prospect of results from the SRS programme in the southern hemisphere becoming available within the next few years, we may regard the problem of the establishment of a fundamental reference frame for proper motions down to about $m=11$ as, in principle, solved. This reference frame, through the large number of stars involved, will be readily accessible to photographic observers, with carte-du-ciel type, and even longer focus telescopes, for the reduction of relative proper motions to an absolute system.

Suitable extra-galactic objects can, in principle, be used as an absolute reference frame, but their uneven distribution over the sky, and scarcity at all but the faintest magnitudes means that they cannot generally be used as a practical reference frame in particular fields. The extreme importance of the programmes now being carried out at Pulkovo, Lick and the Yale southern station, is in the determination of precessional and other errors in the fundamental systems, and in the improvement in our general knowledge of stellar kinematics.

Extrapolation of the fundamental reference frame to fainter magnitudes is dangerous; it will therefore always be necessary to appeal to statistical arguments, based on assumptions about stellar kinematics, to derive absolute proper motions of stars fainter than about $\mathrm{m}_{-\mathrm{pg}}=12$.

The statistical methods of reduction of proper motions to an absolute system have been built up partly from evidence from radial velocities of bright stars, both directly through the solar motion and indirectly through estimates of velocity dispersions for the derivation of mean parallaxes. But radial velocity observations of stars fainter than about the ninth magnitude, in sufficient numbers for general statistical investigations, are not available, and it is all the more important to test that a consistent kinematic picture emerges whenever radial velocity and proper motion data are combined, for example in the derivation of statistical parallaxes of particular objects, for the calibration of absolute magnitudes (e.g. Section 3, below).

Secular parallaxes and solar apex

Secular parallaxes can either be derived from measurement of absolute proper motions, or inferred from mean parallaxes and an assumed solar velocity. The position of the solar apex is not critical for the determination of secular parallaxes, but is of crucial importance for the computation of the components of parallactic motion in a particular field.

Secular parallaxes, as functions of apparent magnitude and galactic latitude, have been derived in two investigations carried out at the Leander 
McCormick Observatory, and have been summarised by Vyssotsky and Williams (1948, p. 35). These are on the FK3 system and include values for mean apparent magnitudes from $m=9$ to 12 .

Deutsch (1942) has determined secular parallaxes on the PGC system, down to $\mathrm{m}_{\mathrm{pg}}=14.5$ from the Pulkovo Catalogue of proper motions in the Selected Apgeas (Deutsch, 1940). Aiso, Binnendijk $(1943$, p. 18) has given relative secular parallaxes from a combination of data in the Pulkovo Catalogue and the Radcliffe Catalogue (Knox-Shaw and Scott Barret, 1934).

There are wide divergencies between Deutsch's and the McCormick secular parallaxes for $\underline{m}_{p g}<11$, but even between $\underline{m}_{p q}=11$ and 13 the average differences McCormick - Deutsch are -!'005, - 9904 and +!'002 in the low, medium and high galactic latitude zones respectively. That these could be due to the difference between the two fundamental systems employed is suggested by the comparison given by Vyssotsky and Williams (loc. cit. p. 23) between the FK3 and GC systems. The run of differences is qualitatively similar to the McCormick-Deutsch differences, as though not quite so large. It seems preferable to disregard the absolute secular parallaxes given by Deutsch, and to use his data to give differential values, in the same way as Binnendijk has done. We thus avoid any uncertainty due to the fundamental system, but are still left with the possibility of magnitude equation affecting the brighter magnitudes.

Recently, Fatchikhin (1968) has published preliminary results of the Pulkovo programme of proper motions measured relative to galaxies, in which he gives a solution for the solar apex and secular parallaxes in three latitude zones, for his reference stars which were in the range $\underline{m}_{p g}=14.5$ - 15. These values of the secular parallaxes can be used to provide the zero point for integrating the differential secular parallaxes given by Binnendijk, and deduced from Deutsch's data, when due allowance has been made for his exclusion of stars with $|\mu|>0 ! 05$. From the statistics of large proper motion stars in the north polar cap, recently published by Luyten (1969), we deduce the corrections !'000, :001 and"002 to Fatchikhin's secular parallaxes in the low, medium and high latitude zones respectively; substantially the same corrections are obtained from the data for large proper motion stars given by Binnendijk (loc. cit. ). The resulting "absolute" secular parallaxes from the data of Deutsch and Binnendijk are plotted as open circles and triangles in Fig. 1. The agreement between the slopes of the two sets of data is satisfactory, but is perhaps not too surprising as they are based partially on common material. For comparison, we give also the mean values of the secular parallaxes from the two McCormick investigations. The agreement between the two entirely independent approaches, in the magnitude range $\underline{m}_{m}=11-13$ is fairly good in the high and low latitude zones, but not quite $\mathrm{gg}$ good in mid-latitudes; however, there is considerable improvement over the original differences between McCormick and Deutsch, particularly in the low latitude zone, which indicates that the procedure adopted here, of fixing the zero point of the differential secular parallaxes by means of Fatchikhin's values, is substantially correct. Recomputation 


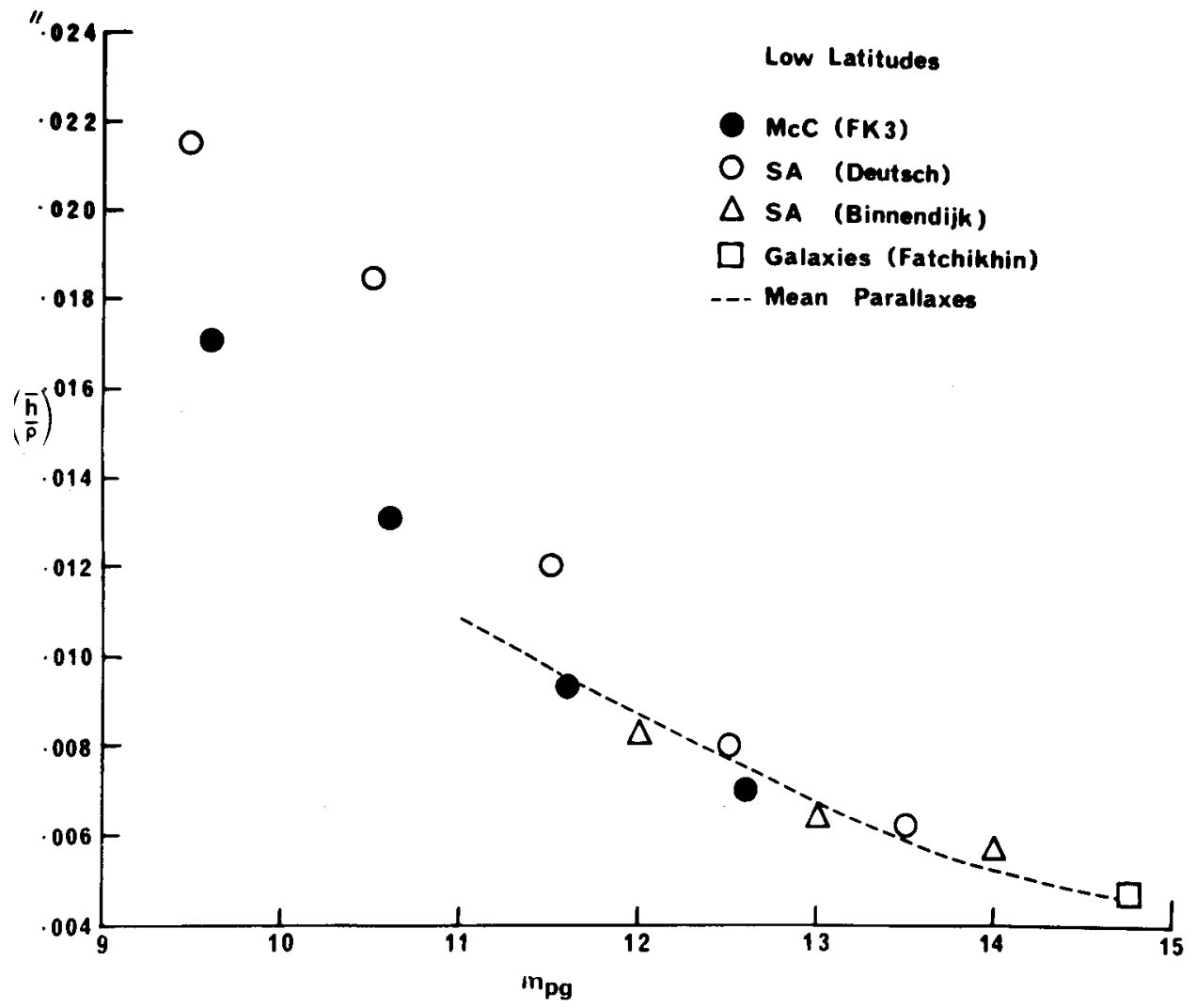

Figure 1a. Secular parallaxes: low latitudes. 


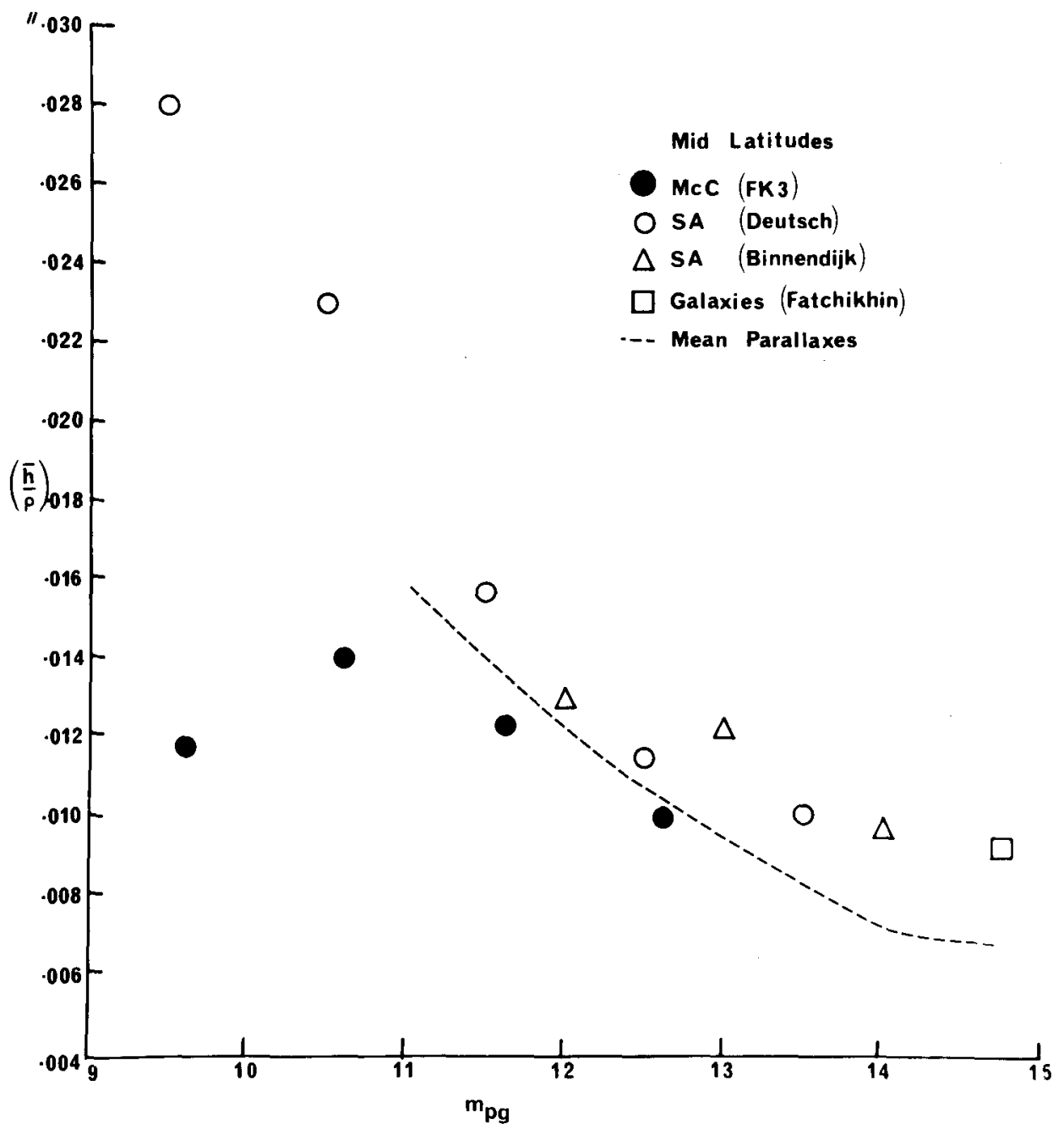

Figure 1b. Secular parallaxes: mid-latitudes. 


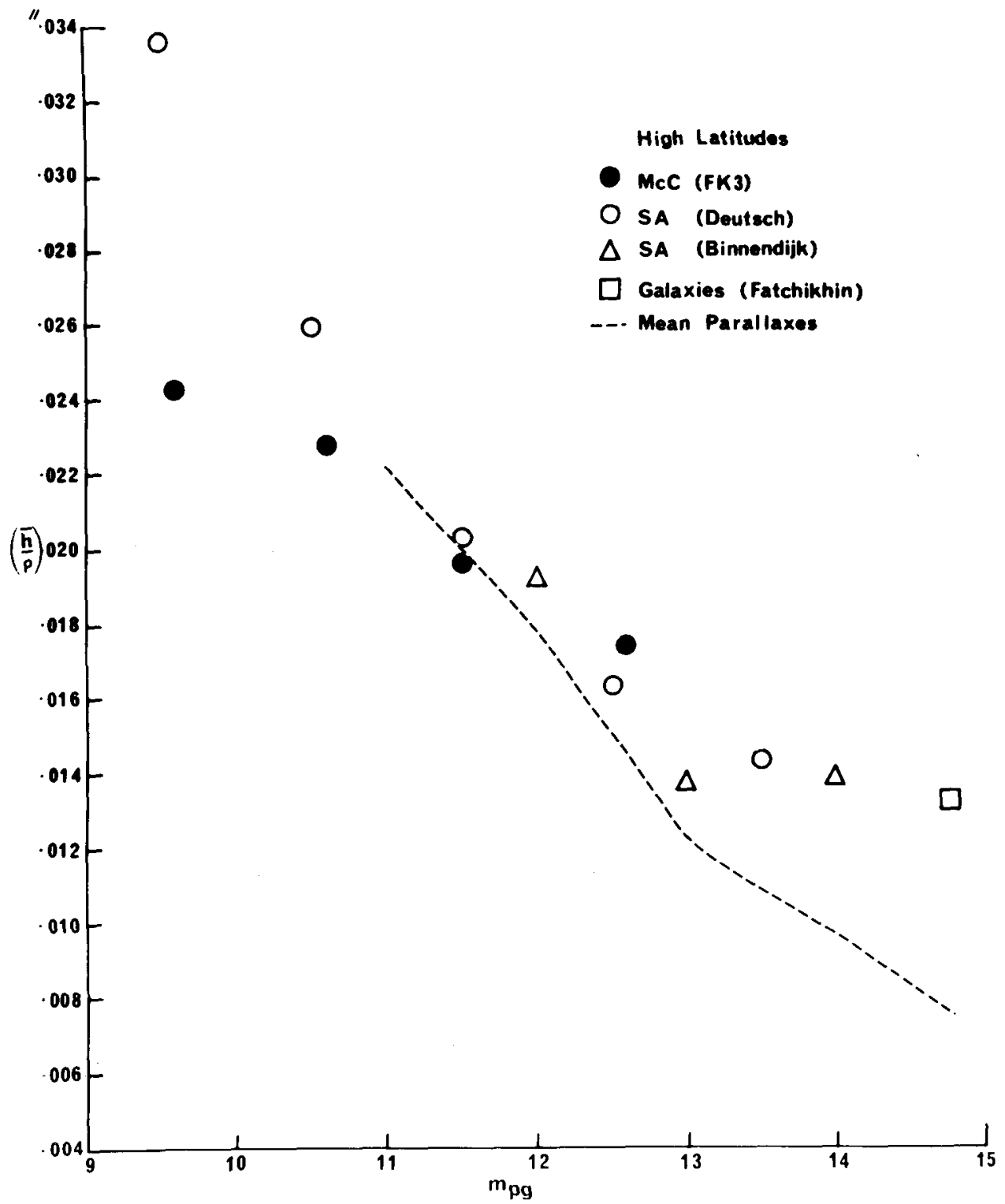

Figure 1c Secular parallaxes: high latitudes. 


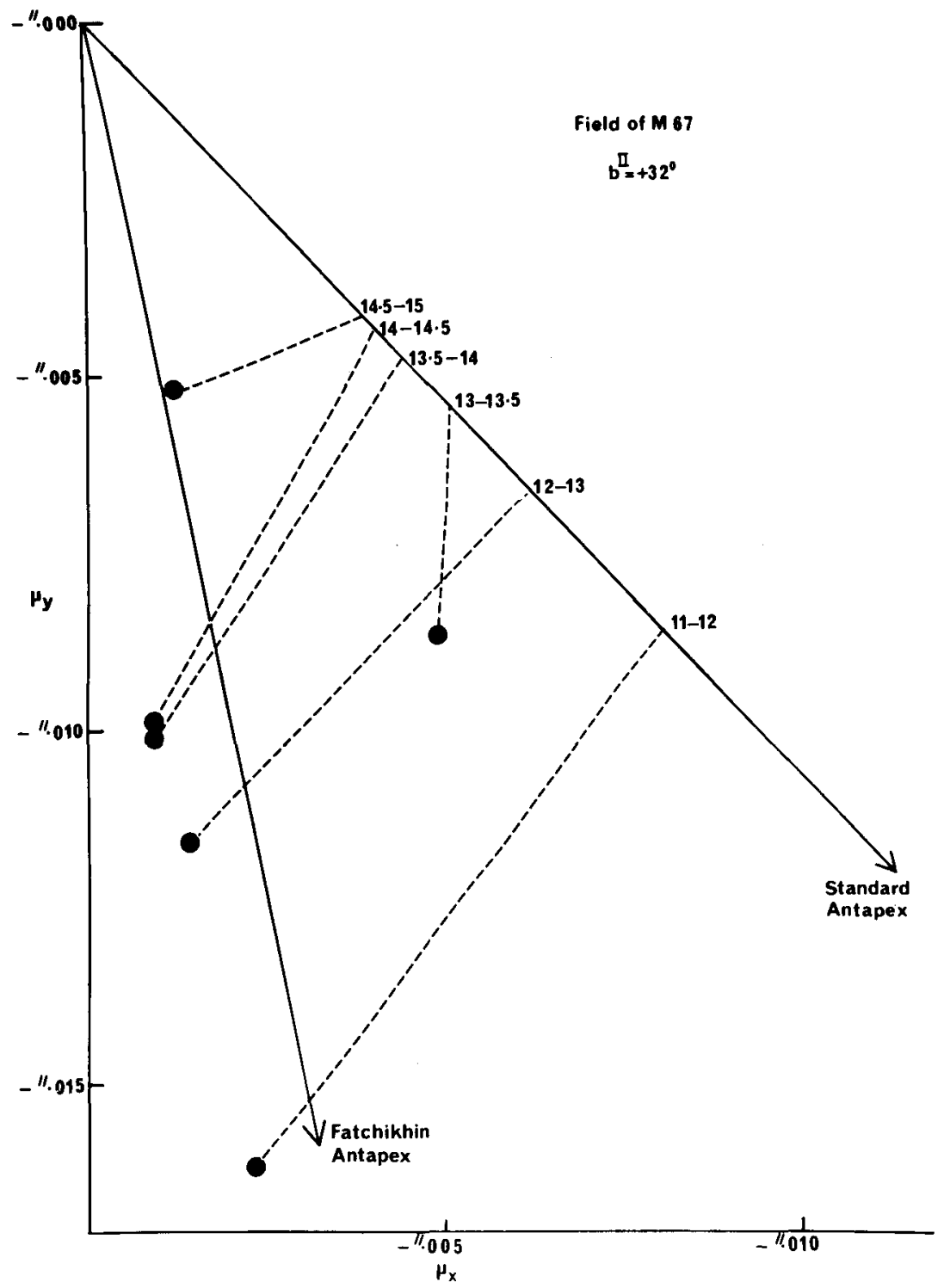

Figure 2. Proper motion vector diagram: Field of M67. 


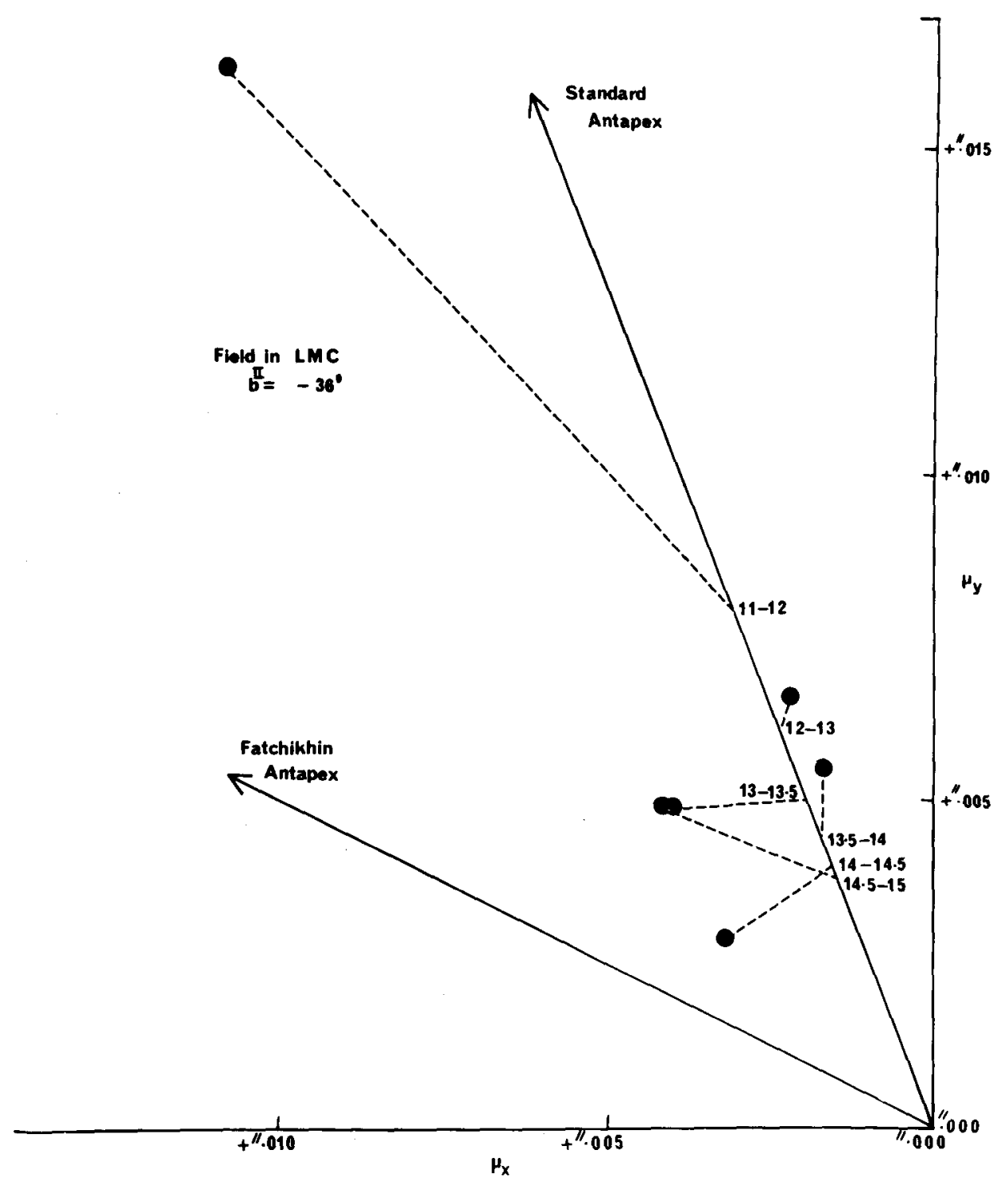

Figure 3. Proper motion vector diagram: Field in LMC. 
of the McCormick values on the FK4 system may well reduce the remaining discrepancies still further. In the magnitude range $m_{p}=9-11$, the differences between Deutsch and McCormick are still seriouts, but as has been remarked above, this could well be due to magnitude equation in the Pulkovo data.

The broken lines in Figure 1 (a), (b) and (c) represent the secular parallaxes inferred from a combination of the assumed solar velocity of 20 $\mathrm{km} / \mathrm{sec}$, and the mean parallaxes deduced by Binnendijk (loc. cit. Table 8 ) from the observed dispersions of proper motions in the selected areas combined with Oort's model of the velocity and luminosity distribution, (Oort, 1936 ). In the low latitude zone, the agreement between the absolute secular parallaxes and those inferred from the mean parallaxes is excellent, indicating that Oort's model must be substantially correct near the galactic plane, or at least, that errors in the solar velocity are compensated by errors in the mean parallaxes. In the higher latitude zones, and for $m$ $>12$, however, there is a tendency for the absolute secular parallaxes to $\mathrm{be}$ larger than those inferred from the mean parallaxes. The discrepancy could in principle be due either to under-estimation of the mean parallaxes or the solar velocity, but such evidence that is available suggests that the latter interpretation is correct.

In discussing the motions and luminosities of tenth magnitude $\mathrm{K}$ stars, Edmondson, Vyssotsky and Janssen (1949) found that the absolute magnitude of the giants in high latitudes, derived from their secular parallax assuming a solar velocity of $20 \mathrm{~km} / \mathrm{sec}$, was over a magnitude fainter than that derived from a comparison between the proper motion dispersion and the velocity dispersion derived from a preliminary discussion of their own radial velocity data. They interpreted this as being due to an under-estimate of the solar velocity relative to these stars, the true value being about $30 \mathrm{~km} / \mathrm{sec}$, and argued that a higher solar velocity relative to stars several hundred parsecs from the galactic plane was only to be expected since such stars would have moderate inclinations and hence larger than average eccentricities, and would on the average show a drift relative to stars in the plane in the direction opposite to that of galactic rotation.

Such an interpretation requires a shift of solar apex, and is confirmed by the direction for the apex found by Fatchikhin, namely

$$
\underline{A}=302: 5 \pm 4: 5 \quad \underline{D}=+32: 6 \pm 2: 7
$$

This apex implies the following ratios for the galactic velocity components of the Sun:

$$
\underline{u}_{\odot}: \underline{v}_{\odot}: \underline{w}_{\odot}::+0.33:+0.94:-0.01
$$

whereas the standard apex $\left(\underline{\mathrm{A}}=270^{\circ}, \underline{\mathrm{D}}=+30^{\circ}\right)$ implies:

$$
\underline{\mathrm{u}}_{\odot}: \underline{\mathrm{v}}_{\odot}: \underline{\mathrm{w}}_{\odot}::+0.51=+0.76:+0.39
$$


Thus Fatchikhin's apex is consistent with a larger $v_{\odot}$ component for his faint high-latitude stars, compared with nearby stars.

If we now assume that the mean parallaxes are correct, we find the value of the solar velocity in intermediate and high latitudes given in Table I, from the absolute secular parallaxes plotted in Figures 1 (b) and (c). In deducing these values, we have resolved Binnendijk's differential secular parallaxes, derived from northern hemisphere fields, to Fatchikin's apex by multiplying them by $\sec 29^{\circ}=1.14$.

\section{Table I}

\section{Solar Velocity $(\mathrm{km} / \mathrm{sec})$}

$\begin{array}{ccc}\text { mpg } & \text { mid latitudes } & \text { high latitudes } \\ 12 & 22 & 23 \\ 13 & 27 & 23 \\ 14 & 27 & 29 \\ 14.7 & 27 & 35\end{array}$

Further confirmation of Fatchikhin's apex can be found in a recent paper by one of us (Murray 1968). In this investigation of field stars in the region of $M 67\left(b^{11}=+32^{\circ}\right)$, average luminosities and photometric parallaxes of stars grouped in small ranges of colour and apparent magnitude were deduced from the observed dispersions of relative proper motions. A zero point of the proper motions, defined as the proper motion corresponding to stars with "zero parallax" was derived from relative parallactic motions. For the present purpose we have regrouped the stars in ranges of $\mathrm{B}$ magnitude, and the mean proper motions relative to "zero parallax", of six magnitude groups are shown in Figure 2. The full lines in the figure represent the directions towards the standard antapex and Fatchikhin's antapex and it is quite evident that most of the plotted points confirm the latter. The calibrated points along the standard antapex line indicates the parallactic motion which would have been expected, using Binnendijk's mean parallaxes and the solar velocity of $20 \mathrm{~km} / \mathrm{sec}$; the dotted lines indicate the errors in the reduction to absolute proper motion if the "expected" parallactic motions had been used. The average transverse velocity components of main sequence stars in this field, with mean $B \approx 14.3$ and mean photometric parallax 0:'00179 have been deduced (loc. cit. p. E353). In combination with Fatchikhin's apex, we find a solar velocity of $27 \pm 9 \mathrm{~km} / \mathrm{sec}$ for these stars, which is in excellent agreement with the value in Table I for this magnitude and latitude zone, in spite of the large standard error. The mean parallax of these stars is also in almost exact agreement with the value given by Binnendijk (loc. cit. Table 8 ) for this magnitude, in the latitude zone $30^{\circ}$ to $45^{\circ}$ and is independent confirmation of the corrections 
of the mean parallaxes, since we suspect very few giants at this magnitude.

However, somewhat contradictory evidence appears from a discussion of proper motions in a region of the Large Magellanic Cloud (R.O. B. 66, $1963)$, which is centred in a southern latitude $\left(\underline{b}^{11}=-36^{\circ}\right)$. Figure 3 shows similar data to Figure 2, for the foreground stars in this field. In this case the zero point has been taken to be the LMC itself, and the plotted points have been corrected for first order galactic rotation and differential rotation using the standard formulae, and $A=+15 \mathrm{~km} / \mathrm{sec} / \mathrm{kpc}$ and $B=-10 \mathrm{~km} /$ $\mathrm{sec} / \mathrm{kpc}$. In this case the standard antapex; if the latter were correct for this field, the transverse velocity of the LMC would have to be about 600 $\mathrm{km} / \mathrm{sec}$ which seems unlikely.

Table II gives the secular parallaxes observed in the M67 and LMC fields, obtained by using both the standard (S) and Fatchikhin's ( F) apices. In both fields $\mathrm{N}$ is the number of stars included in each group, and the number in brackets the number of large proper motion stars $(|\mu|>0: 1)$ which have been excluded. The secular parallaxes inferred from Binnendijk's mean parallaxes and the solar velocity of $20 \mathrm{~km} / \mathrm{sec}$, for the mid latitude zone (Figure 1(b)), are given under "Binn", and the absolute secular parallaxes, based on the average of Deutsch and Binnendijk in the mid latitude zone ( Figure $1(\mathrm{~b})$ ) are given under "Abs." in Table II.

Table II

\section{Observed Secular Parallaxes}

\begin{tabular}{ccccccccc} 
B & \multicolumn{3}{c}{ M67 Field } & \multicolumn{7}{c}{ LMC Field } \\
& S & F & $\underline{N}$ & S & F & $\underline{N}$ & Binn. & Abs. \\
$11-12$ & .0163 & $: 0228$ & 23 & $: 0387$ & .0294 & $15(1)$ & $: 0140$ & $: 0157$ \\
$12-13$ & .0115 & .0163 & $41(1)$ & .0111 & .0072 & $39(2)$ & .0108 & .0120 \\
$13-14$ & .0102 & .0137 & $66(1)$ & .0095 & .0070 & $75(3)$ & .0082 & .0106 \\
$14-15$ & .0072 & .0104 & 130 & .0081 & .0072 & 122 & .0069 & .0093
\end{tabular}

In the LMC, Fatchikhin's apex clearly gives unsatisfactory results, while those computed using the standard apex are in much better accordance with the absolute values. The bright group in this field must be a freak; of the 15 stars included, 5 have proper motions exceeding 0:04, in the same direction, and the remainder are all less than 0!'025. This is perhaps an extreme case of the sampling errors which are likely in the statistical reduction to an absolute system, when only a few reference stars are used. In the M67 field the secular parallaxes derived from the standard apex are in good agreement with the absolute values, but, as we have already seen (Figure 2), the observed proper motions are more consistent with Fatchikhin's apex which leads to much higher secular parallaxes. This suggests that the absolute values may themselves have been underestimated by the 
use of an inappropriate solar apex, and raises the question whether we are in fact justified in assuming a unique apex for all magnitudes and latitudes.

Proper motions and radial velocities of RR Lyrae stars

The apparent tendency of the mean parallaxes of faint stars at medium and high galactic latitudes to produce underestimates of their secular parallaxes, when combined with the standard solar velocity is also evident in an entirely independent investigation of the kinematics of halo RR Lyrae variables by one of us (Clube and Jones; in preparation). In this investigation, the kinematic characteristics of these stars), as derived fromradial velocities are compared with those derived from proper motions which have been reduced to an absolute system by kinematic methods using reference stars whose mean magnitude is about $m=12$. The self-consistency of the radial velocity and proper motion data is $\mathrm{pg}$ xpressed by the determination of their total likelihood, which is found to be very sensitive to even a moderate correction to the secular parallaxes of the reference stars. The average secular parallax, based on the number count technique, using Binnendijk's mean parallaxes and the standard solar velocity of $20 \mathrm{~km} / \mathrm{sec}$ was found to be :013. The effect of arbitrary corrections to the component of the Sun's velocity in the direction of galactic rotation, have been examined, and the results given in Table III. In the Table, $v_{0}$ is the component of the Sun's velocity in the direction of galactic rotation, and $(h / \rho)$ the corresponding secular parallax of the reference stars assuming $u$ values corresponding to the standard solar motion. The remaining columns give the natural logarithm of the total likelihood referred to an arbitrary zero-point. In $L$, the average rotational velocity of the $R R$ Lyrae stars relative to the Sun, $\mathrm{v}_{\mathrm{RR}}$, the velocity dispersion of the RR Lyrae stars in the $\underline{u}$ direction $\sigma\left(\underline{u}_{R R}\right)$ and the variance of their absolute magnitudes $\sigma_{M}$. The first line of Tâble III corresponds to the solution depending on the value of the secular parallax deduced from number counts and the standard solar motion; the values of $\underline{v}_{R_{R}}$ and $\sigma\left(\underline{u}_{R R}\right)$ from this solution show considerable discordance from the corresponding values, -209 and $124 \mathrm{~km} / \mathrm{sec}$, which are derived from radial velocities alone.

Table III

Motions of RR Lyrae variables

$\begin{array}{cccccc}\begin{array}{c}\mathrm{v} \\ \mathrm{km} / \mathrm{sec}\end{array} & \underline{\mathrm{h}} / \rho & \text { In } \underline{\mathrm{L}} & \begin{array}{c}\mathrm{v}_{\mathrm{RR}} \\ \mathrm{km} / \mathrm{sec}\end{array} & \sigma\left(\underline{\mathrm{u}}_{\mathrm{RR}}\right) & \sigma^{2} \\ 15 & : 013 & -5.6 & -179 \pm 5 & 175 \pm 7 & 0.50 \\ 26 & .019 & -1.3 & -187 & 153 & 0.20 \\ 37 & .025 & +1.0 & -191 & 141 & 0.02 \\ 48 & .032 & +1.5 & -194 & 139 & -0.01 \\ 59 & .039 & +1.0 & -194 & 140 & -0.02\end{array}$


Since negative values of $\sigma_{M}{ }^{2}$ are not physically possible, the run of $\ln \underline{L}$ and of $\sigma_{M}{ }^{2}$ both point to a secular parallax of about :022 as being the most appropriate for the reference stars. Part of the difference between this figure and 0:013 could be attributed to inadequate allowance having been made for high proper motion stars, or a systematic overestimation of the number counts used in estimating the mean parallaxes, but it is unlikely to account for it all. The corresponding value of $\mathrm{v} \rho$ is about $+31 \mathrm{~km} / \mathrm{sec}$; since the assumed value of $\underline{u}_{-}$is $+10 \mathrm{~km} / \mathrm{sec}$, from the standard solar motion, the ratio $\mathrm{u}_{-}: \mathrm{v}_{-\odot}$ agrees ${ }^{-}$well with that given by Fatchikhin's position for the solar apex.

Requirements for improved kinematic reduction methods.

The reduction of relative proper motions to an absolute system, according to established practice, consists of three parts:

(i) the differential galactic rotation of the centroid of the reference stars, relative to the local centroid defined by some average of the stars near the Sun, referred to axes rotating with the local stars around the galactic centre.

(ii) parallactic motion, due to motions of the Sun and the reference stars relative to their respective centroids.

(iii) the absolute rotation of the axes defined by the local stars.

The differential rotation of the centroids is conventionally described by the simple formula $2 \mathrm{Ax}$, where $\mathrm{x}$ is the coordinate of the centroid of the reference stars relative to the lofal ceptroid (assumed to coincide with the Sun, in space) in the direction $\underline{1}^{11}=\underline{b}=0$, which is based on the simple shear model with cylindrical symmetry.

To take account of the absolute rotation, it is necessary to assume a value for $A-B$, the angular velocity of the local centroid around the galactic centre. This can be derived from proper motions which have been referred to a well defined fundamental system (e.g. Fricke 1967) or to a reference frame based on extra-galactic objects, provided that an adequate model of their relative motions is available.

The division into differential rotation, or shear motion, and parallactic motion is a matter of convention. Variations in the direction of the solar apex, and the apparent change in solar velocity with distance from the plane, which have been discussed in section 2, may be regarded as being due to failure of the simple shear law $2 \mathrm{~A} x$ to represent adequately the total drift of any but the youngest objects of Population I relative to the local centroid. There is no reason to suppose that such deviations from the simple shear model depend only on galactic latitude, or distance; there may well be large scale anomalies affecting any region of the Galaxy, which have been concealed hitherto by the drastic smoothing procedure adopted in the classical treatment of secular parallaxes and solar apex. The apparent contradictions between the M 67 and LMC field stars (Figures 2 and 3 ) may be indications of such anomalies. 
It has been shown elsewhere (Clube 1967) that the standard error of a reduction to absolute proper motion, carried out according to current methods is unlikely to be less than about $\pm: " 005$. If higher precision is required, then it will be necessary to measure many more reference stars than is customary, and to carry out a detailed study of their likely luminosity and kinematic characteristics from their colours and relative proper motions. In addition, a model of general kinematics as a function of position in the Galaxy, which is much more realistic than the simple shear model, must be developed.

\section{Observational needs}

The current programme for the improvement of proper motions in the northern Selected Areas, which is being undertaken at Herstmonceux, will provide some of the data needed for the solution of the problems discussed above. (see e.g. Contopoulos 1970). The large amount of astrometric data will yield relative proper motions with accidental errors of about \pm :001 from which it will be possible to derive accurate values for the proper motion dispersions. For direct reduction to an absolute system, we shall rely on proper motions relative to galaxies; through the kind cooperation of $\mathrm{Dr}$. Vasilevskis, some $30-40$ suitable stars in each area will be measured as part of the Lick programme. AGK 3 stars can of course be measured in each area also, but their images are large on the plate exposed to $\underline{m}_{\mathrm{pg}} \approx 15$, and there is serious possibility of magnitude errors.

This astrometric programme, by itself, is not enough. First epoch plates for proper motions in the southern Selected Areas have been taken with the Yale-Columbia telescope (Plaut 1968). It is highly desirable that these plates should be repeated, in order to give whole-sky coverage.

All proper motions should be supported by good two or three colour photometry, in default of objective-prism spectra for the faint stars. No firm plans have so far been made for obtaining the photoelectric standards in each area, which will be needed to provike colours and magnitudes for the faintest stars, in the programme; however, a recent publication by Purgathofer (1969), provides excellent data in seven areas in the galactic anticentre and north pole regions, and these areas are being measured first in the Herstomonceux astrometric programme.

Finally, it may not be superfluous to appeal once again for radial velocity observations of faint stars in sufficient numbers to provide statistical data on the variation of solar motion and velocity dispersion in different parts of the sky. It is to be hoped that the detailed results of the programme of Edmondson, Vyssotsky and Janssen (loc. cit.) will be published soon, and that someone, somewhere will be willing to undertake systematic radial velocity observations on a similar scale to at least the eleventh magnitude.

We are grateful to Mr. Z. Aslan for many useful discussions on the topics discussed in this paper. 
Binnendijk, L. , 1943. B. A. N. 10, 9

Clube, S. V. M. , 1967. Q. F.R. A.S. 7, 257

Contopoulos, G., 1970. Thessaloniki Contributions No. 53, 114

Deutsch, A. N., 1940. Pulkovo. Pub, (Series II) Vol. LV.

Deutsch, A. N., 1942. Pulkovo. Bull, 138, 55

Edmondson, F. K., Vyssotsky, A. N., and Janssen, E. M. , 1949. Ap. J. 110,182

Fatchikhin, N. V. , 1968. Soviet Astronomy - A. J. 12, 289

Fricke, W. , 1967. A. J. 72, 1368

Knox - Shaw, H. , and Scott Bennett, H.G., 1934. The Radcliffe Catalogue of Proper Motions in the Selected Areas 1 to 115. (O. U. P.)

Luyten, W. J., 1969. Proper Motions Survey with the Forty - eight Inch Schmidt Telescope XX (University of Minnesota)

Murray, C. A., 1968. R. Obs. Bull. 141

Dort, J.H., 1936. B, A, N, 8, 75

Plaut, L. , 1968. Trans. I. A. U. XIIIB, 187

Purgathofer, A. Th. , 1969. Lowell Obs. Bull. No. 147

Vyssotsky A. N., and Williams, E.T. R., 1948. Pub. Leander McCormick Observatory, $\mathrm{X}$.

\section{DISCUSSION}

Luyten: I also have a private method of correcting relative proper motions to absolute values. When I did the motions of the Humason - Z wicky stars and the Feige stars and all these other faint blue stars, I took the then accepted values of the secular parallax and multiplied them by 1.5 , because I was working in high galactic latitudes. You see I had a good education from Hertzsprung. His famous dictum was that anyone who wanted to be successful in photometry had to be somewhat of a charlatan, and I applied this to proper motion work. Since you mention several values of the apex I might add one thing. At the moment I am busy on a magnum opus blinking a seventeen year interval pair of plates on the North American Nebula at 21 hours and $+42^{\circ}$. And the proper motions of the stars there are not really large. I am sure I am getting quite a number of stars with motions of only two seconds per century, hence the region must be very close to at least somebody's apex.

Fricke: How far away are these halo stars according to Clube? And how many are there with no radial velocities?

Murray: Well this is off the record. Its absolute magnitude comes out to be 1. 3 , and about fifty or sixty stars I think it is. All halo, and delta is greater than five. All northern stars. 
Eichhorn: You said you reduced these motions to absolute by a private method. Is that the work which was published on M67 in the Quarterly Journal?

Murray: No. Sorry about the M67 field; that is my private one in the RA Bulletin. It was a study of the detailed characteristics of the stars; I had good two-color photometry for all the stars, and studied the velocities of the proper motion dispersions for the small ranges of color and magnitude to deduce which were the main sequence stars. And then one gets photometric parallaxes for those, and uses those as a reference system. This works very well, and it appears that the zero point must be about right.

Wesselink: Could you say something more about how Fatchikhin determined his apex?

Murray: As I understand it, he got that from a simple solution for the secular parallax. This is against the full curve of programming against galaxies, 14.5 to 15 th magnitude. These are his reference stars. And I do not really see how they could be wrong. There should be no systematic error.

Wesselink: No, but in itself it is a surprising thing that his apex deviates so much from the usual one.

Murray: I am not so sure. Because now we are talking about distant stars and, of course, high latitude stars. And it is well known, of course, that you would expect the velocity, meaning the motion, to be different in high latitudes. In other words, the simple sine law might fit in the plane for Population I objects, but it certainly is not cylindrically symmetrical. I think this is an entirely reasonable result.

van de Kamp: Murray's paper reminded me of my very first paper which I wrote forty-seven years ago which dealt with secular parallaxes of faint stars and being young, I did not conclude the paper on the modest note as you did. I should like to say one more thing. Two months ago I really discovered where the apex is, it is a little town in North Carolina. There is a little sign there saying "Apex." 